\title{
Optical fiber ring resonator characterization by optical time-domain reflectometry
}

\author{
A. Küng, J. Budin, L. Thévenaz, and Ph. A. Robert \\ Metrology Laboratory, Swiss Federal Institute of Technology (École Polytechnique Fédéral de Lausanne), CH1015 Lausanne, Switzerland
}

Received September 4, 1996

\begin{abstract}
A novel method for characterization of optical fiber resonators by an optical time-domain reflectometry (OTDR) technique is reported. This easy-to-use technique yields accurate results for cavity lengths ranging from a few meters to several kilometers. A simple relationship is established between the round-trip cavity loss and the position where the OTDR signal is maximum. The value obtained for the round-trip cavity loss turns out to be quite insensitive to uncertainties in the determination of the OTDR maximum position. (C) 1997 Optical Society of America
\end{abstract}

Optical fiber ring resonators have been widely used in passive and active gyroscopes, ${ }^{1,2}$ narrow-linewidth Brillouin lasers, ${ }^{3}$ optical frequency-selective filters, ${ }^{4}$ and a wide range of optical fiber sensors. ${ }^{5}$ Most of these applications require a high-finesse cavity, so it is of prime importance to know the quality of the closed-loop fiber resonator. The quality and the finesse depend on only two ring parameters ${ }^{6}$ : the intensity transmission coefficient $k$ of the coupler (coupling coefficient $1-k$ ) and the cavity feedback coefficient $k_{r}$ (cavity round-trip loss $1-k_{r}$ ). The basic experimental problem results from the impossibility of measuring the light circulating in the cavity without increasing the intracavity loss and perturbing the resonator operation. One usually characterizes a fiber ring resonator by measuring its intensity transfer function, using a coherent light source. The two parameters $k$ and $k_{r}$ can be determined from this transfer function by measurement of the finesse $F$ of the resonator and the residual intensity transmission $T_{\min }$ at resonance. ${ }^{6,7}$ If the coupler coefficient $k$ is known, measuring either $F$ or $T_{\min }$ is sufficient for determining $k_{r}$. However, this method suffers from several drawbacks: (i) the polarization into the loop must be controlled so that light propagates in a polarization eigenstate, (ii) either the length of the cavity or the wavelength of the light source must be varied to permit scanning of the entire coherent transfer function, and (iii) the linewidth of the light source must be at least 1 order of magnitude smaller than the resonance width $d \nu$ to resolve this resonance properly. The last-named condition can be a seriously limiting factor for long cavity length.

A novel nondestructive technique is proposed that uses an optical time-domain reflectometry (OTDR) instrument to characterize optical fiber ring resonators. The ring structure has the unique feature of letting the OTDR pulse circulate in the ring while generating Rayleigh backscattering. The Rayleigh backscattered light also circulates in the ring but in the opposite direction. One part of the scattered light keeps circulating into the ring, whereas the other part is directed through the coupler onto the detection stage. This re- circulation of the OTDR pulse combined with the recirculation of the Rayleigh backscattered light allows different Rayleigh contributions to be summed and detected simultaneously. Each contribution corresponds to a definite number of turns for the OTDR pulse and a definite scattering point and a definite number of turns for the Rayleigh backscattered light. A typical OTDR trace of a ring resonator is shown in Fig. 1.

The trace is made of equal-length steps corresponding to one round trip in the ring and resulting from the addition of contributions circulating in the ring for a given propagation time. The first steps are given by a few contributions of great intensity. As the step order gets higher the number of contributions grows and their intensity decays, resulting in a maximum whose position depends on the ring parameters.

The aim of the following demonstration is to show that the relevant ring parameters can be determined only from the position of this maximum. For this purpose a model describing the Rayleigh backscattered intensity at the ring output is developed. Problems of the source polarization and the ring birefringence are addressed and lead to a simple equation characterizing the ring. Finally measurements are compared with the model, showing excellent agreement with theory.

According to the schematic description in Fig. 2, the electric field that results from the OTDR probe

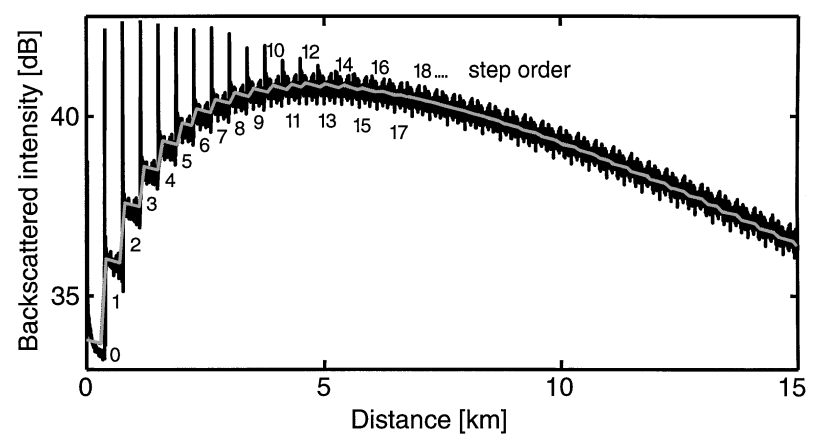

Fig. 1. OTDR trace of a 765-m-long fiber ring resonator with coupler coefficient $k=95 \%$ together with the numerical fit of Eq. (4) below, giving a calculated cavity feedback coefficient $k_{r}=89.5 \%$. 


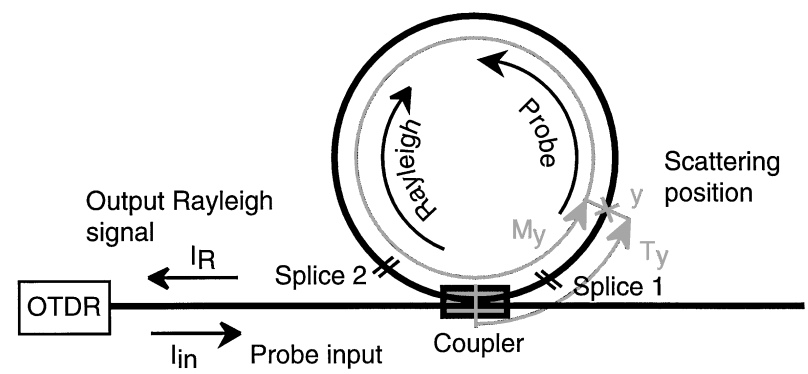

Fig. 2. Schematic description of the measurement technique. The optical paths corresponding to the Jones matrices $M_{y}$ and $T_{y}$ are represented.

pulse propagating $n$ counterclockwise turns in the ring, which is scattered at a distance $y$ from the coupler and then propagates $m$ clockwise turns, is given by

$$
\begin{aligned}
\mathbf{E}_{n m}(y)= & E_{00}(y)(k \xi)^{(n+m / 2)} \exp [-\alpha(n+m) L / 2] \\
& \times \exp [i \beta(n+m) L] T_{y}{ }^{T} M_{y}{ }^{T m} M_{y}{ }^{n} T_{y} \hat{e},
\end{aligned}
$$

where $E_{00}(y)$ corresponds to the electric field amplitude for $n=m=0$ :

$$
E_{00}(y)=\sqrt{I_{\text {in }} R(y)} \xi \exp (-\alpha y) \exp (i 2 \beta y),
$$

where $I_{\text {in }}$ is the input intensity, $\beta$ is the propagation constant, $k$ is the coupler intensity coefficient, $\xi$ is the intensity transmission coefficient of the ring excluding the fiber linear loss, $R(y)$ is the intensity Rayleigh backscattering coefficient, $\alpha$ is the intensity linear attenuation of the fiber, $L$ is the ring length, and $y(0 \leq y \leq L)$ is the distance along the fiber from the coupler to the scattering point. $\hat{e}$ is the Jones vector of the incident light, and $M_{y}$ and $M_{y}{ }^{T}$ are the Jones matrices in the probe (counterclockwise) direction and the backscattered direction (clockwise), respectively, for a full round trip in the ring starting from point $y$ :

$$
M_{y}=\left[\begin{array}{cc}
\cos \theta_{y} \exp \left(i \varphi_{y} / 2\right) & \sin \theta_{y} \exp \left(i \varphi_{y} / 2\right) \\
-\sin \theta_{y} \exp \left(-i \varphi_{y} / 2\right) & \cos \theta_{y} \exp \left(-i \varphi_{y} / 2\right)
\end{array}\right]
$$

where $\varphi_{y}$ and $\theta_{y}$ are the phase delays for a full round trip that are due to linear and circular birefringence, respectively. $T_{y}$ and $T_{y}{ }^{T}$ are the Jones matrices from the coupler to scattering point $y$ for the probe pulse and the Rayleigh backscattered signal, respectively.

The cavity feedback coefficient $k_{r}$ is given by $k_{r}=$ $\xi \exp (-\alpha L)$. We obtain the resulting OTDR trace by summing over $m$ and $n$ each contribution of Eq. (1) that experiences equal propagation times. As these contributions are generated simultaneously by the same source and are also detected simultaneously, they experience the same propagation delay and must be added coherently. A simple intensity summation results in a low-accuracy model. ${ }^{8}$ However, the contributions scattered from different centers are not correlated ${ }^{9}$ and must be added incoherently as a result of the random character of Rayleigh scattering. For a given propagation time each contribution may be scattered by two different centers spaced by a distance $L / 2$, as shown in Fig. 3 for step 1.

These considerations result in the following discontinuous steplike backscattered intensity function:

$$
\begin{aligned}
I_{u}(y)= & I_{0}(y)(k \xi)^{u-1} \exp (-\alpha u L) \\
& \times\left[k \xi\left|\sum_{p=0}^{u}\left(M_{y}^{T}\right)^{u-p}\left(M_{y}\right)^{p} \hat{e}\right|^{2}\right. \\
& \left.+\left|\sum_{p=0}^{u-1}\left(M_{y+L / 2}^{T}\right)^{u-p-1}\left(M_{y+L / 2}\right)^{p} \hat{e}\right|^{2}\right],
\end{aligned}
$$

where $I_{0}(y)=\left|E_{00}(y)\right|^{2}$ correspond to the intensity function for step zero, the integer $u=\operatorname{int}(z / L)$ is the step order, and $z$ is the total propagation distance. Position $y(0 \leq y \leq L / 2)$ of the scattering center is simply related to the other quantities by $z=u L+2 y$. The matrices $T_{y}$ and $T_{y}{ }^{T}$ cancel, as they are identical for each contribution of each coherent summation.

Let us show now that the polarization of the incident light and the ring birefringence have only an amplitude scaling effect on the OTDR signal waveform; the intensity ratio between distinct steps remains constant.

Using the unimodularity property $\operatorname{det}\left(M_{y}\right)=1$ and a simple but tedious calculation give

$$
\left|\sum_{p=0}^{u}\left(M_{y}^{T}\right)^{u-p}\left(M_{y}\right)^{p} \hat{e}\right|^{2}=\lambda\left(\theta_{y}, \varphi_{y}, u\right),
$$

where $\lambda\left(\theta_{y}, \varphi_{y}, u\right)$ is a scalar function of the birefringence parameters $\theta_{y}$ and $\varphi_{y}$ and the step order $u$ and is independent of the incident polarization $\hat{e}$. Substituting Eq. (5) into the intensity function [Eq. (4)] for both summations results in a backscattered intensity $I_{u}(y)$, independently of the input light polarization.

To analyze the effect of birefrigence on the signal waveform one must address short and long rings separately. For short rings let us assume that over one round trip the birefrigence is independent of the wavelength and that the circular birefringence results in a phase delay much smaller than $\pi$. This condition limits the cavity length to $\sim 10$ times the birefringence beat length. In this case low birefringence delay makes possible the following first-order approximation:

$$
\lambda\left(\theta_{y}, \varphi_{y}, u\right) \cong F\left(\theta_{y}, \varphi_{y}\right) \lambda(0,0, u)=F\left(\theta_{y}, \varphi_{y}\right)(u+1)^{2},
$$

which results from summation (5) with no birefringence and where $F\left(\theta_{y}, \varphi_{y}\right)$ is a scalar function of the birefringence parameters. For a fixed reflection at point $y$ the birefringence dependence of the signal is identical for any steps except step zero, which has a

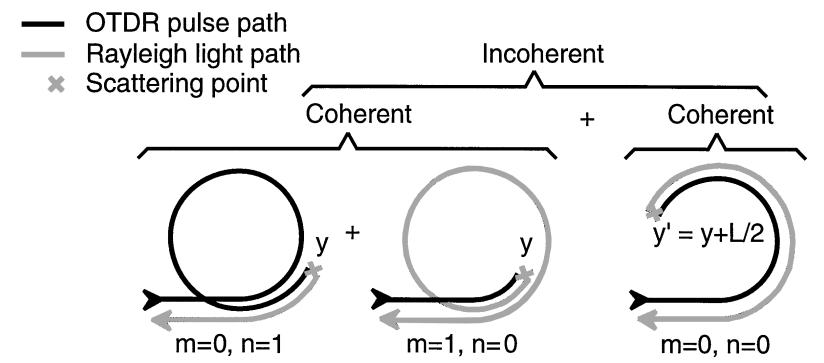

Fig. 3. Addition of the three contributions of Step 1 . The contributions scattered at the same point are added coherently. 
Table 1. OTDR Measurements of Several Fiber Ring Resonators of Increasing Length Compared with Measurements with a Coherent Light Source ${ }^{a}$

\begin{tabular}{lcccc}
\hline & \multicolumn{2}{c}{ OTDR Measurements } & & \multicolumn{2}{c}{ Coherent Measurements } \\
\cline { 2 - 3 } Ring Characteristics & $Z_{\max }(\mathrm{m})$ & $k_{r}(\%)$ & & $T_{\min }(\%)$ \\
\hline$k=94.8 \%, L=5.568 \mathrm{~m}$ & $136 \pm 1.5$ & $97.19 \pm 0.09$ & $9 \pm 1$ & $97.21 \pm 0.1$ \\
$k=96.9 \%, L=10 \mathrm{~m}$ & $445 \pm 3$ & $98.66 \pm 0.04$ & & $13 \pm 1$ \\
$k=90.3 \%, L=10 \mathrm{~m}$ & $165 \pm 2$ & $98.10 \pm 0.13$ & & $95 \pm 1$ \\
$k=97.1 \%, L=26.5 \mathrm{~m}$ & $490 \pm 5$ & $92.42 \pm 0.1$ & $19 \pm 1$ & $98.06 \pm 0.05$ \\
$k=95.0 \%, L=765 \mathrm{~m}$ & $9440 \pm 150$ & $89.5 \pm 0.23$ & - & - \\
\hline
\end{tabular}

${ }^{a}$ Experimental conditions could not be achieved for a coherent measurement on the long fiber ring.

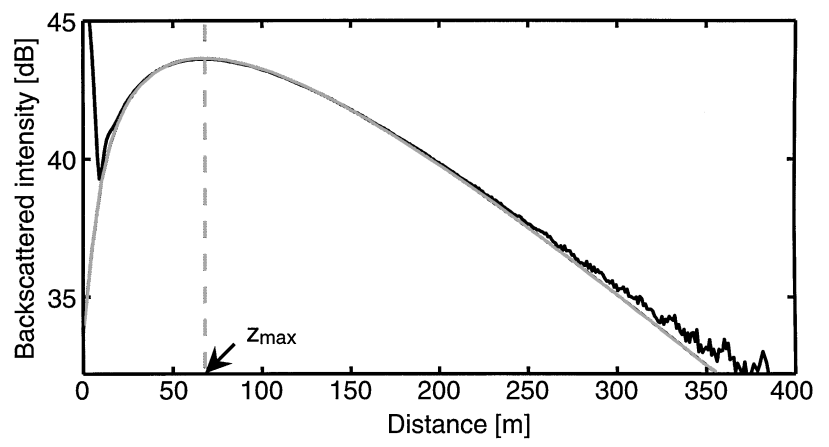

Fig. 4. OTDR trace of a 5.568-m-long fiber ring resonator with coupler coefficient $k=94.8 \%$ together with the numerical fit of Eq. (7), giving a calculated cavity feedback coefficient $k_{r}=97.2 \%$.

single scattering contribution and thus shows no interference. When step zero is discarded the normalized OTDR waveform does not therefore depend on birefringence. In the ideal case in which the birefringence is purely linear the whole signal becomes independent of the birefringence as $M_{y}=M_{y}{ }^{T}$. For long rings the birefringence contains large linear and circular components, so the birefringence delay depends on the wavelength, and because OTDR sources have wide spectra this results in an ensemble average on polarization delays. The signal is averaged and becomes independent of the birefringence.

This analysis demonstrates that the polarization changes only the level of the global signal without altering its shape, provided that either the short- or the long-cavity assumption is satisfied. So one can use the model without considering polarization by discarding step zero.

The summations contained in the steplike backscattered intensity function [Eq. (5)] can be replaced by the corresponding integrals, resulting in a continuous function:

$$
\begin{aligned}
I(u)= & I_{\mathrm{in}} R(u) \xi^{2} \exp \left[-\alpha L\left(u+\frac{1}{2}\right)\right] \\
& \times(k \xi)^{u-1}\left[k \xi(u+1)^{2}+u^{2}\right]
\end{aligned}
$$

where $u$ is now a continuous parameter:

$$
u=(z / L-1 / 2) \text {. }
$$

Differentiation of Eq. (7) over $z$ followed by a firstorder approximation yields a simple approximate relation between the position $z_{\max }$ of the maximum backscattered intensity and the resonator parameters:

$$
k k_{r}=\exp \left(-\frac{2 L}{z_{\max }}\right)
$$

Thus, if $k$ and $L$ are known, we determine $k_{r}$ simply by measuring the position $z_{\text {max }}$ where the Rayleigh backscattered signal is maximum. The particular relationship between $z_{\max }$ and $k_{r}$ results in a high accuracy of $k_{r}$, even if the relative accuracy of $z_{\max }$ is poorer. Table 1 compares $k_{r}$ determined by this method with $k_{r}$ derived by the conventional measurement that uses a coherent source for different fiber ring resonators.

Figure 1 shows an OTDR trace obtained from a long ring resonator and the numerical fit of relation (4). Each round trip is clearly resolved. Reflection peaks at the output fiber end are measured, but they are discarded before fitting.

Figure 4 shows the OTDR trace of a short ring resonator and its corresponding fit obtained by use of the continuous function [Eq. (7)]. In this case the OTDR resolution is not sufficient to resolve each step, but the information can still be obtained, which is a remarkable feature of the method.

This simple method demonstrates that long and short optical fiber ring resonators can be successfully characterized by use of an OTDR, even for a cavity shorter than the OTDR spatial resolution. The accuracy of standard OTDR instruments is sufficient for reliable measurements of the intracavity loss of a fiber ring resonator.

\section{References}

1. K. Takiguchi and K. Hotate, IEEE Photon. Technol. Lett. 4, 7 (1992).

2. F. Zarinetchi, S. P. Smith, and S. Ezekiel, Opt. Lett. 16, 6 (1991).

3. L. Thevenaz, J. Boschung, and P.-A. Robert, Electron. Lett. 30, 18 (1994).

4. K. Kalli and D. A. Jackson, Opt. Lett. 17, 17 (1992).

5. Y. Ohtsuka and Y. Imai, Appl. Opt. 24, 4199 (1985).

6. L. F. Stokes, M. Chodorow, and H. J. Shaw, Opt. Lett. 7, 6 (1982)

7. F. Zhang and J. W. Y. Lit, J. Opt. Soc. Am. A 5, 8 (1988).

8. D. J. Ives, R. L. Palmer, and B. Walker, Electron. Lett. 27, 4 (1991).

9. P. Gysel and R. K. Staubli, J. Lightwave Technol. 8, 4 (1990). 